Math. Model. Nat. Phenom.

Vol. 5, No. 1, 2010, pp. 203-223

DOI: $10.1051 / \mathrm{mmnp} / 20105109$

\title{
Individual Cell-Based Model for In-Vitro Mesothelial Invasion of Ovarian Cancer
}

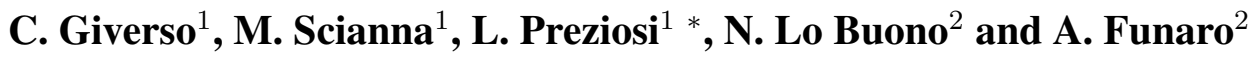 \\ ${ }^{1}$ Department of Mathematics, Politecnico di Torino, Corso Duca degli Abruzzi 24 \\ 10129 Torino, Italy \\ ${ }^{2}$ Laboratory of Immunogenetics, Department of Genetics, Biology and Biochemistry, \\ University of Turin Medical School, Via Santena 19, 10126 Torino, Italy
}

\begin{abstract}
In vitro transmesothelial migration assays of ovarian cancer cells, isolated or aggregated in multicellular spheroids, are reproduced deducing suitable Cellular Potts Models (CPM). We show that the simulations are in good agreement with the experimental evidence and that the overall process is regulated by the activity of matrix metalloproteinases (MMPs) and by the interplay of the adhesive properties of the cells with the extracellular matrix and between cells, both of the same type and of different types. In particular, the process depends on the ability of the cell to induce the loosening of cadherin-mediated junctions. Coherently with experiments, it is found that single cell invasion is more conservative with a crucial role played by MMPs. A similar important role is played in cell spheroid invasion, which in comparison is more disruptive. It achieves monofocal or multifocal characteristics according to the relative adhesion affinity among cells or between them and the mesothelial layer.
\end{abstract}

Key words: ovarian cancer metastasis, mesothelium, cellular potts model, hybrid model AMS subject classification: 92C17, 92C37, 92C50

\section{Introduction}

Ovarian cancer (OvCa) is the fifth leading cause of tumor-related deaths in the Western world (with little change in its incidence in recent decades) [2], the second most common gynecological

\footnotetext{
${ }^{*}$ Corresponding author. E-mail: luigi.preziosi@polito.it
} 
carcinoma and the leading cause of death from gynecological malignancies, since its mortality rate is high, compared to other cancers [4]. Significant contributors to its high mortality rate are the vague or absent symptoms in the early stages of the disease, the lack of reliable tumor markers and the recurrences, generally fatal, despite good initial responses to chemotherapy. For these reasons, long-term survival is rare since $70 \%$ of the patients presents at diagnosis extensive and widespread intraperitoneal dissemination throughout the abdomen and the pelvis [6].

The majority $(\approx 90 \%)$ of ovarian cancer stems from surface epithelium which overlies the ovary [9].

Epithelial ovarian cancer metastasization (a crucial process in the growth of a cancer, that enables the primary tumor mass to proliferate and spread to distant sites) involves exfoliation of tumor cells as single cells or aggregates from the primary tumor into the abdominal cavity, and the successive implantation on and invasion through the mesothelial lining of the peritoneum [1]. Seeding into the peritoneal cavity is frequently associated to ascites formation. Such a transmigration is generally defined as the process of a cell entering or leaving a biological membrane (or layer) and it is essentially a physiological mechanism used by specialized cells to move and reach distant organs: cancer cells pathologically implement transmigration to create colonies of secondary tumors.

Clinically, ovarian cancer progression is divided into 4 stages by the Federation Internationale de Ginecologie et d'Obstretique (F.I.G.O.) based on tumor spreading. In the early stage (stage I) the disease is confined to one or both the ovaries, while in stage II it has begun spreading, with localized extensions into the adjacent pelvic tissues and organs. As the disease progresses into stage III, the tumor has spread to the upper abdominal cavity, until at the final stage IV the metastatic tumor reaches distant, extra-peritoneal sites.

In this work we model typical in vitro experiments reproducing the principal biological aspects of the ovarian cancer transmesothelial migration. In particular, we focus on the differences between the invasion of single cells and of cell aggregates or spheroids. The simulation system integrates: (a) a Cellular Potts Model (CPM), developed for instance in [13, 15, 21, 24, 32, 35], and reviewed in $[20,30]$, that captures mechanisms of cellular adhesion and motion and the degradation of the extracellular matrix (ECM), both the one that surrounds the mesothelium with a pericellular layer and the one between the mesothelium and the Petri dish, (b) a continuous model for the diffusion and uptake of chemotactic factors due to the presence of ECM molecules and the mesothelial layer, (c) a continuous model for MMPs release from tumor cells and relative activation. All the simulations are performed with a modified and adapted version of CompuCell3 ${ }^{\dagger}$ package $[14,16,28]$. In particular, we have implemented different plug-ins, each of them code a specific biological behavior (e.g., matrix fiber degradation).

The results of this work are in good agreement with experimental evidence, mimicking all the described features, and let understand the importance of the role in the ovarian cancer transmigration process of the different families of adhesion molecules (i.e., cadherins and integrins). In particular, it is demonstrated that a crucial role in the transmigration of single cells is played by MMPs [4, 18], while the adhesion affinity among tumor cells and mesothelial cells is crucial to determine the inclusion of the tumor cell in the mesothelial layer [27].

\footnotetext{
${ }^{\dagger}$ http://www.compucell3d.org
} 
In addition to the previous phenomena in the case of the invasion of an aggregate, the relative adhesion affinity between the tumor cells and between them and the mesothelium determines whether the invasion is monofocal or multifocal. In any case, it is found, in agreement with experiments, see Figure 1, that single cell invasion is more conservative [34], while the invasion of tumor spheroids causes the disruption of the mesothelium, see Figure 2, with the appearance of tumor foci which infiltrate the peritoneum and potentially seed metastatic tumor growth [10, 37].
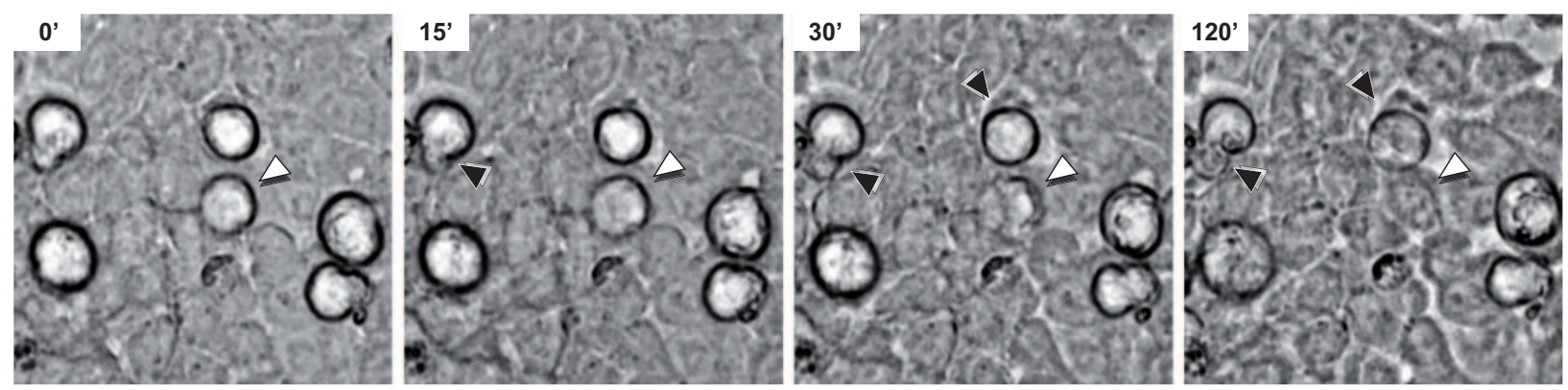

Figure 1: OvCa transmesothelial migration was visualized by real-time microscopy using non-malignant Met-5A mesothelial cells grown on fibronectin. Nomarski differential interference contrast (DIC) images were captured using an F-View II CCD camera connected to an Olympus IX70 inverted microscope controlled by Cell ${ }^{R}$ imaging software (Olympus Biosystems, Milano, Italy). Samples were placed on a heated stage (Thermoplate, Tokai Hit, Olympus Biosystems) set at $37^{\circ} \mathrm{C}$ and images were taken using a 40x objective. OvCa cells were added on mesothelial monolayer, and frames were captured every 15 seconds for 2 hours. Frames corresponding to 0, 15, 30 or 120 minutes of the movie are shown. The migration of a single cell (white arrows) through a mesothelial cell junction is appreciable without damage of the mesothelial layer. At the end of tumor cell transmigration (120 minutes) the junction is restored. After 30 minutes, a number of cells in the same field started to migrate (black arrows).

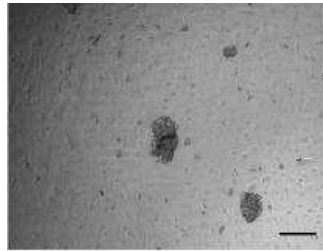

(A)

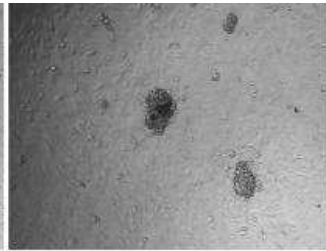

(B)

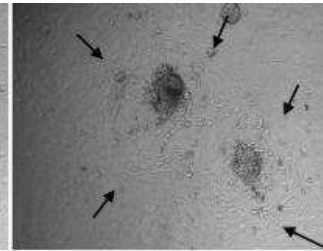

(C)

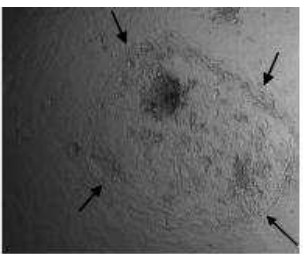

(D)

Figure 2: Spheroid dissemination and mesothelial monolayer invasion assay: spheroids (5-10/well) were seeded onto the HPMC and digitally photographed at (A) $1 \mathrm{~h}$ after plating $(\mathrm{t}=0),(\mathrm{B}) 1$ and (C) 3 and (D) 7 days.

\section{Phenomenological Description}

After detaching from the ovarian capsule the original malignant cells shed from the primary tumor into the peritoneal cavity, where they disseminate transported by the peritoneal fluids and ascites, until they find attachment sites for further growth and begin to establish secondary tumors, often 
without the need to enter the vasculature. The ascites, present in almost one third of ovarian cancer patients, is a generally voluminous exudative fluid, containing selected cell populations mainly consisting of tumor cells, lymphocytes and mesothelial cells and it facilitates tumor cell transport throughout the abdominal cavity [31]. The neoplastic cells in the ascites are present either as single cells, as aggregates, or as multi-cellular spheroids. Their migration is also regulated by environmental factors such as ECM components, cytokines, growth factors or chemotactic factors, mainly secreted by mesothelial cells [26].

In the current model of ovarian cancer spread, tumor cells are able to survive and subsequently to attach to and to invade through the mesothelium, an epithelial-like monolayer [25] that lines the organs of the abdominal cavity and is the elective site of ovarian carcinoma disaggregation, dissemination and metastatic outgrowth [40]. The successful metastatic process, governed by the biophysical properties of cancer cells combined with the remodeling of intra- and inter-cellular proteins, that regulate cell-cell adhesion (for example, cadherins) and cell-ECM interactions (for example, integrins), can be divided in two main steps: i) adhesion of the tumor cells to the mesothelial layer and ii) invasion of the mesothelial layer.

\subsection{Single Cell Transmigration}

Transmesothelial migration has been reproduced in vitro by plating an ovarian cancer cell line (NIH: OVCAR-3) on a non-malignant transformed human mesothelial cell layer (Met-5A), grown on an extracellular matrix protein as in Figure 3. Experimental evidence showed that single ovarian tumor cells adhesion to the monolayer is mainly mediated by the interactions between $\beta 1$-integrin and some of its epitopes [38] and selected ECM proteins (such as laminin, fibronectin, vitronectin, and type I and IV collagen). ECM proteins are secreted by the mesothelial cells and form a sort of pericellular matrix around the layer. CD44-hyaluronan and E-cadherins also interact with the mesothelial cells [19].

The subsequent transmigration across the mesothelium requires the activity of selected Matrix Metalloproteinases (MMPs), endopeptidases that predominantly degrade any structural components of the ECM, along with a variety of cell adhesion molecules [42]. They also affect the relative cellular signalling pathways and functions and control cell migration. Moreover, the MMPs are involved in the release of cell-membrane-bound precursors of many growth factors, whose receptors are MMP substrates. They can cleave and activate their own zymogen forms [8]. In pathological conditions, the MMPs actively contribute to cancer progression: clinical data suggest that benign tumor cells acquire malignant properties following up-regulation of MMP expression and that, conversely, highly malignant cells become less aggressive when MMP activity is reduced.

In ovarian cancer transmigration assay, the over-expression of $\beta 1$-integrins results in the downregulation of the E-cadherin function and in the activation and co-localization of MMP-2, MMP-9, MMP-14 and MT-1 MMP, which also cleave CD44 molecules, releasing their extracellular domain [11]. Finally, the activity of the secreted MMPs promotes cancer invasion by cleaving and regulating the normal function of the N-cadherins, which are the predominant cell-cell adhesion molecules holding the mesothelial cells together: the brokage of these bonds causes the retraction of the mesothelial layer at the attachment site of malignant cells, opening the way for the 


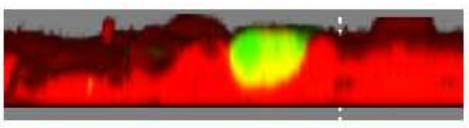

(A)

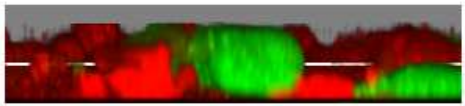

(D)

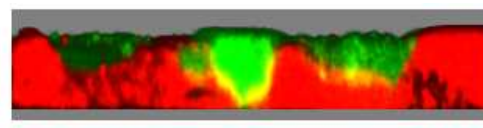

(B)

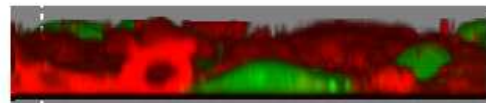

(E)

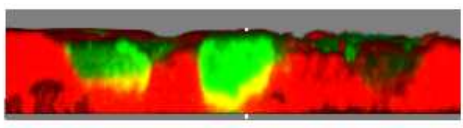

(C)

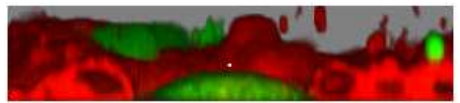

(F)

Figure 3: Transmesothelial migration assay: non-malignant transformed human pleural mesothelial cells (HPMCs), Met-5A cells were labeled with the PKH26GL cell linker kit (colored in red), seeded on coverslips coated with fibronectin $(10 \mu \mathrm{g} / \mathrm{mL})$ and grown until confluence at $37^{\circ} \mathrm{C}$. The human ovarian carcinoma cell line NIH:OVCAR-3 was stained with $5 \mu \mathrm{M}$ CFSE 20 minutes at $37^{\circ} \mathrm{C}$ (colored in green). CFSE-labeled tumor cells $\left(1.5 \times 10^{5}\right)$ were plated on HPMCs and incubated for 3 hours at $37^{\circ} \mathrm{C}$. Non-adherent OvCa were carefully removed. Samples were analyzed by sequential scanning of the XY planes recorded along the Z-axis (step size: $1.5 \mu \mathrm{m}$ ) and then processed using the 3-dimensional reconstruction software bioView3D and visualized as orthogonal views. In (A) a cell is on the top of the mesothelial layer. In (B) and (C) cells are inducing opening in the layer and passing through. In (D) a cell is in the middle of the layer. In $(\mathrm{E})$ and $(\mathrm{F})$ cells crossed the mesothelial layer and the cells re-joined closing the gaps previously created over the malignant cells.

subsequent invasion [29], as shown in Figures 3B-D. Some data suggest that the mesothelial cells themselves may produce low levels of MMPs and induce MMPs expression by ovarian cancer cells [41]. Other data have shown that ovarian tumor cells secrete the urinary-type plasminogen (uPA), which participates in the conversion of plasminogen to plasmin and allows further amplification of sub-mesothelial ECM degradation, and other serine proteases. However, uPa and serineproteases may play a less important role than MMPs in facilitating cancer invasion.

Upon complete migration of cancer cells across the mesothelium, the normal function and signalling of N- and E- cadherins are recovered [33], as the mesothelial cells re-join closing the gaps previously created over the migrated malignant cells [39], as shown in Figures 3E-F.

\subsection{Multicellular Spheroid Invasion}

In the ascites of ovarian cancer patients, tumor cells also exfoliate as aggregates or multicellular spheroids, whose most important function is to create an anchorage-independent in vivo tumor microenvironment, supporting mechanisms of cell survival and growth through homotypic cell-cell adhesion [37]. This function largely depends on the expression, the activity and the large variety of selected adhesion molecules, including cadherins, integrins, ECM proteins, glycosaminoglycans, and proteoglycans within the spheroids, which appear dramatically different compared to the cells growing isolated or as a monolayer [3].

Because of their low proliferative rate, spheroids are resistant to therapies directed against fastgrowing tumor cells, to some forms of chemotherapy and to apoptosis induced by radiation or by conventional therapeutic drugs [5]; they also have altered kinetics of drug absorption. These evidences suggest that spheroids may represent a tenacious and important intermediate for secondary 


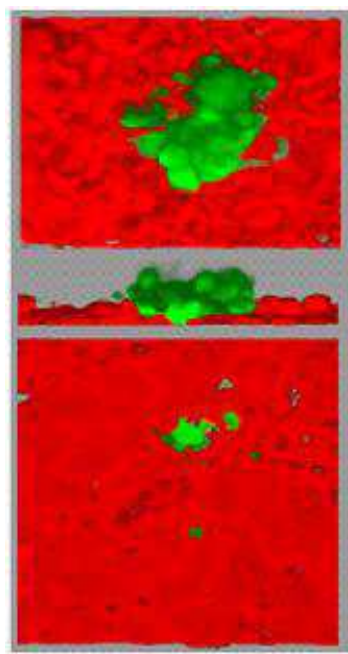

(A)

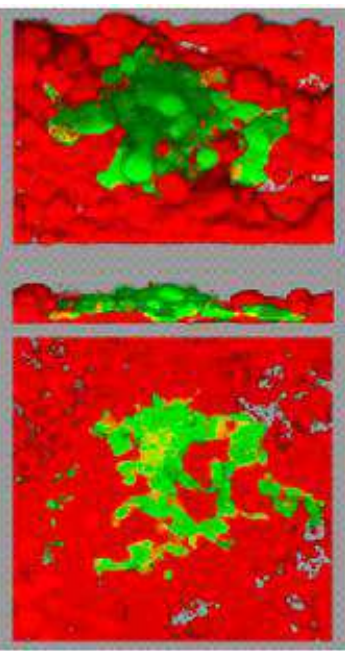

(B)

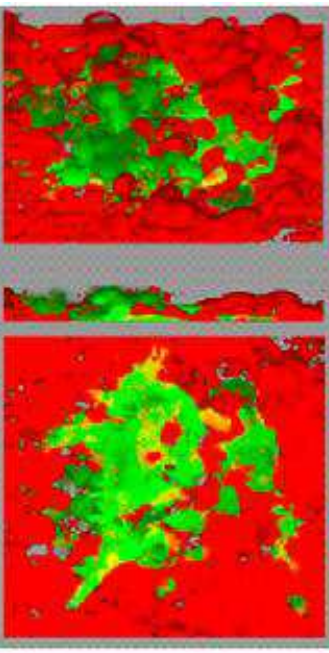

(C)

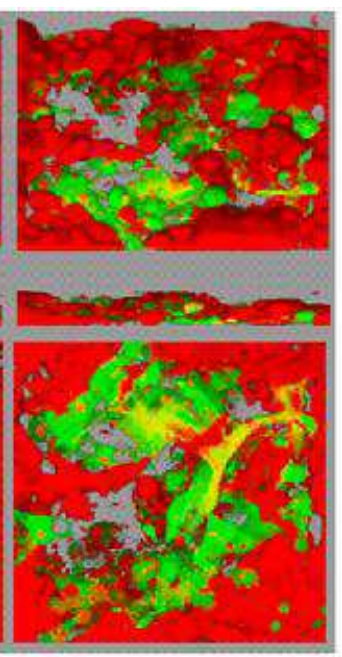

(D)

Figure 4: HPMCs $\left(2 \times 10^{5}\right)$ were labeled with PKH26GL (red), seeded on fibronectin coated coverslips and grown until confluence at $37^{\circ} \mathrm{C}$ (as described in Figure 3). Spheroids were generated using a modification of the hanging droplet method [17]. Briefly, cells were stained in green and then placed (50 cells/15 $\mu$ l of RPMI with $15 \%$ FCS) on the cover of a tissue culture dish, and the cover was placed over a dish containing PBS to prevent dehydration of the hanging droplets. After 4 days, spheroids were seeded on HPMCs layer and allowed to adhere for 3 hours before imaging. Images show the $3 \mathrm{D}$ reconstruction of the same field visualized from the upper, transverse and bottom side at time zero (A), $24 \mathrm{~h}(\mathrm{~B}), 48 \mathrm{~h}(\mathrm{C})$ and $72 \mathrm{~h}(\mathrm{D})$.

tumor growth and an accurate model system of ovarian metastasis [36].

The initial formation of ovarian spheroids is strongly regulated by $\beta 1$-integrin subunits [7], but it is also mediated by compensatory mechanisms, through other cell-cell interactions, such as gap junctions, tight junctions and desmosomes [43] and the activity of cadherins and different ECM components (including fibronectin, laminin, and type I and IV collagen) incorporated into the spheroid from the ascites fluid.

To experimentally test their ability to disseminate and their metastatic potential, spheroids were generated in vitro and seeded onto a mesothelial monolayer, generally anchored to an ECMsubstrate, see Figure 4. The spheroid dissemination, defined as the tumor mass spreading on top of the monolayer without forming invasive foci, requires the interaction of $\beta 1$-integrins with the ECM proteins secreted by the mesothelial cells, with the contribution of other adhesion molecules. The quantified overall adhesion levels of ascites spheroids are somewhat lower than those reported for isolated carcinoma cells, which possibly reflects a change in cell adhesive ability upon acquisition of the spheroid morphology.

The process of invasion, defined as the establishment of proliferating foci of ovarian tumor cells within the same focal plane as the layer, starts with the retraction of some mesothelial cells (Figure 4A). Suddenly, the extracellular matrix molecules underlying the monolayer become exposed, allowing the spheroids to grow forming large foci of invasion, which appear to grow laterally, almost within the same plane of the layer (Figure 4B). The area occupied by the original spheroids pro- 
gressively increases in size, as the mesothelial cells recede and are overtaken and replaced by the malignant mass (Figures 4C-D). The invasion is regulated by the proteases secreted and activated by the tumor cells. Some experimental data also indicate that ovarian cancer spheroids secrete much greater amounts of both pro-MMP-2 and MMP-9 compared to cells grown as a monolayer, and that both MMPs are present in the active form $([5,11]$ and AF, personal observation).

Quantitative studies evaluating the percentage of invasion demonstrate that tumor spheroids plated in the same well do not exhibit the same invasive properties, despite sharing an identical environment, since only a proportion of them establishes foci of invasion. These data imply that the metastatic potential is not merely induced by the microenvironment, but likely relies on physiological differences in the invasive characteristics of every single spheroid.

\section{Mathematical Model}

The Cellular Potts model (CPM) is a grid-based 3D multiscale mathematical model, which uses an hybrid approach to describe subcellular, cellular and tissue level interactions, combining cellular automata and continuum methods. The CPM uses a lattice to describe cells and associates an integer index (or spin) $\sigma$ to each lattice site (or voxel) $\mathbf{x} \in \mathbb{R}^{2}$ to identify the spatial extent and localization of each cell at any instant. A domain (i.e. collections of contiguous lattice sites with the same index) represents a cell. For example, Figure 5 shows three 2D cells, which require three distinct indices. Thus, a collection of $\mathrm{N}$ cells is described by defining $\mathrm{N}$ spins, $\sigma(\mathbf{x})=1,2, \ldots, \mathrm{N}$, and different cells (with different Ids) could belong to the same cell type $\tau$. Cell-cell contacts occur through adjacent voxels which belong to different cells. Some cellular behaviors are employed in the lattice, but others, which have diffusive dynamics, require the introduction of macroscopic models that lead to an hybrid environment [22].

Instead of directly representing the forces that cause cells to rearrange, the CPM aggregates them into an effective energy, the Hamiltonian $H$, which contains true energies (e.g., cell-cell adhesion) and terms that mimic energies (e.g., the response of a cell to chemotactic and haptotactic gradients), all described within the same structure, and whose gradient is the force acting at any point in the pattern. The pattern evolves under strong damping to stochastically reduce its energy. We implement the cells' membrane fluctuations driving toward a global system energy minimization (rather than to one of multiple local minima) using the Metropolis algorithm for Monte Carlo Boltzmann dynamics: we randomly pick a lattice site $\mathbf{x}$, attempt to copy its spin $\sigma(\mathbf{x})$ into a randomly chosen neighbor $\mathbf{x}^{\prime}$ (for better isotropy we use the 20, first to four nearest neighbors, see Figure 5) and use the acceptance function:

$$
P(\Delta H)= \begin{cases}e^{\frac{-\Delta H}{k T_{\tau}}} & \Delta H>0 ; \\ 1 & \Delta H \leq 0 ;\end{cases}
$$

where $\Delta H$ is the net energy difference of the system configuration before and after the copy, $T_{\tau}$ is an effective Boltzmann temperature, defined as the intrinsic motility for each type of cells and 


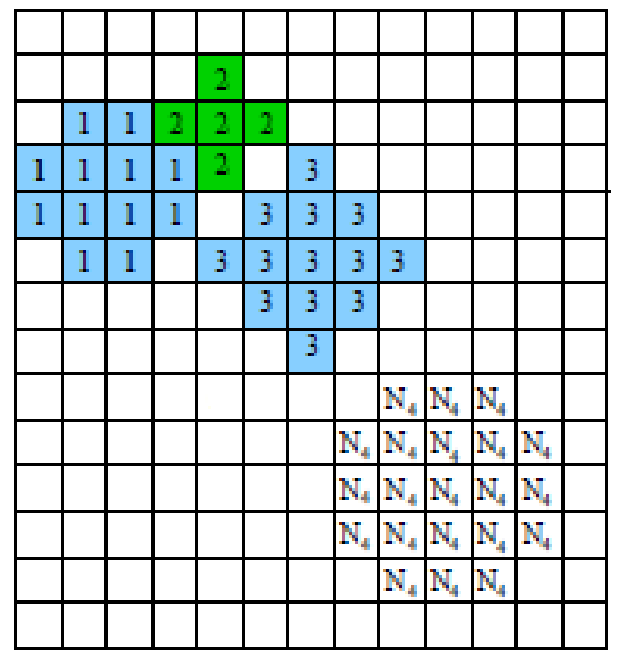

Figure 5: The CPM grid and representation of cells. The shading denotes the cell type. Different cells (e.g. cells 1 and 3) may be of the same cell type. We also show the fourth neighbour interactions of pixel S on a two-dimensional grid.

related to the size of their typical fluctuation (and it has nothing in common with the actual tissue temperature) and $k$ is a constant converting $T_{\tau}$ into units of energy. One iteration of the method is termed a Monte Carlo Step (MCS) and comprises $n$ copy attempts, where $n$ is the number of lattice sites in the system domain.

A typical effective energy $H$ includes cell volume and surface constraints, terms for adhesion and for responses to chemical stimuli:

$$
H=H_{\text {shape }}+H_{\text {adhesion }}+H_{\text {chemical }} \text {. }
$$

A cell of type $\tau$ has an experimentally prescribed target volume $\left(V_{\tau}\right)$ and surface area $\left(A_{\tau}\right)$. Translating actual volumes (respectively, surfaces) to CPM target volumes (respectively, surfaces) involves fixing the ratios between the CPM lattice size in voxel and the actual biological domain length. The volume and the surface of each simulated cell fluctuate around these relative target values, due to changes in osmotic pressure, pseudopodial motion and depending on the membrane elasticity, cytoskeletal properties and incompressibility: $H$ enforces these targets by exacting an energy penalty for deviations with the terms

$$
H_{\text {shape }}=H_{\text {volume }}+H_{\text {surface }}=\lambda_{\tau}\left(V_{\sigma}-V_{\tau(\sigma)}\right)^{2}+\gamma_{\tau}\left(S_{\sigma}-S_{\tau(\sigma)}\right)^{2},
$$

where $V_{\sigma}$ and $S_{\sigma}$ are the current volume and surface of cell $\sigma, \lambda_{\tau}$ and $\gamma_{\tau}$ are the Lagrangian multipliers related, respectively, to volume and surface cell elasticity (i.e., cells' resistance to compression). Cell growth (respectively, death) can be modeled by allowing the values of $\mathrm{V}_{\tau}$ and $\mathrm{S}_{\tau}$ to increase (respectively, decrease) with time, but in the other cases they are fixed.

$H_{\text {adhesion }}$ describes the net adhesion/repulsion between two cell membranes: it is the product of the total area of contact and the binding energy per unit area $J_{\tau, \tau^{\prime}}$, which depends on the spe- 
cific properties of the interacting cells, such as the level of the expression of certain cell adhesion molecules. The form of $H_{\text {adhesion }}$ is:

$$
H_{\text {adhesion }}=\sum_{\mathbf{x}, \mathbf{x}^{\prime}} J_{\tau(\sigma(\mathbf{x})), \tau\left(\sigma\left(\mathbf{x}^{\prime}\right)\right)}\left(1-\delta_{\sigma(\mathbf{x}), \sigma\left(\mathbf{x}^{\prime}\right)}\right),
$$

where $\mathbf{x}, \mathbf{x}^{\prime}$ represent two neighboring lattice sites and the Kronecher delta is $\delta_{x, y}=\{1, x=$ $y ; 0, x \neq y\}$, ensuring that only the links between surface sites in different cells contribute to cells' adhesion energy. Since the CPM has a minimization philosophy, negative $J$ 's model strong adhesion, while high positive $J$ 's are related to weak adhesion forces. The adhesive interactions operate over a prescribed range around each lattice site (see Figure 5).

Cells can also respond to chemical signals by moving along diffusible or substrate-bond concentration gradients of signal molecules. The first mechanism is called chemotaxis, the second haptotaxis. Such biological behaviors require a representation of the evolving and spatially varying chemical concentration field and a model mechanism linking this field to the CPM lattice framework [23]. We implement the preferential motion of a cell in the direction of a chemical gradient by including in the Hamiltonian $H$ the term:

$$
H_{\text {chem }}=\sum_{\mathbf{x}} \mu_{\tau} h(\mathbf{x})
$$

that leads, for linear response (higher order response would be also possible) to the extra reduction of energy at the time of copying

$$
\Delta H_{\text {chem }}=\sum_{\mathbf{x}, \mathbf{x}^{\prime}} \mu_{\tau}\left(h(\mathbf{x})-h\left(\mathbf{x}^{\prime}\right)\right),
$$

where $\mu_{\tau}$ is an effective potential related to the cell type $\tau, h$ is the local concentration of the chemical substance, whose evolution in time and in space is macroscopically described, and $\mathbf{x}$ and $\mathbf{x}^{\prime}$ are, respectively, the source and the target site of the copy attempt.

\section{Simulation of Single Cell Invasion}

In our simulations the transmesothelial migration of a single ovarian cancer cell is modeled as the two dimensional $162 \mathrm{px} \times 50 \mathrm{px}$ section illustrated in Figure $6(\approx 162 \mu \mathrm{m} \times 50 \mu \mathrm{m}$, since in our lattice 1 px corresponds to $\approx 1 \mu \mathrm{m}$ ), where the border at the bottom is the virtual Petri dish, labeled as $\tau_{s}$. The mesothelial layer is formed by $27 \mathrm{px} \times 9 \mathrm{px}$ rectangular cells $\left(\tau_{m}\right)$, and surrounded by a sort of pericellular matrix constituted by ECM components $\left(\tau_{e}\right)$. The area between the simulated mesothelium and the dish is a mixture of interstitial fluid $\left(\tau_{f}\right)$ and ECM molecules ( $\tau_{e}$ again), that mimics the experimental sub-mesothelial-type matrigel used to anchor the monolayer. The virtual ECM components are inelastic and initially arranged in random lines, representing fiber bundles. The round cancer cell $\left(\tau_{c}\right)$, with a diameter of $14 \mathrm{px}$, is placed in proximity of the layer, within the peritoneal fluid (generalized lattice sites $\tau_{f}$ ). 


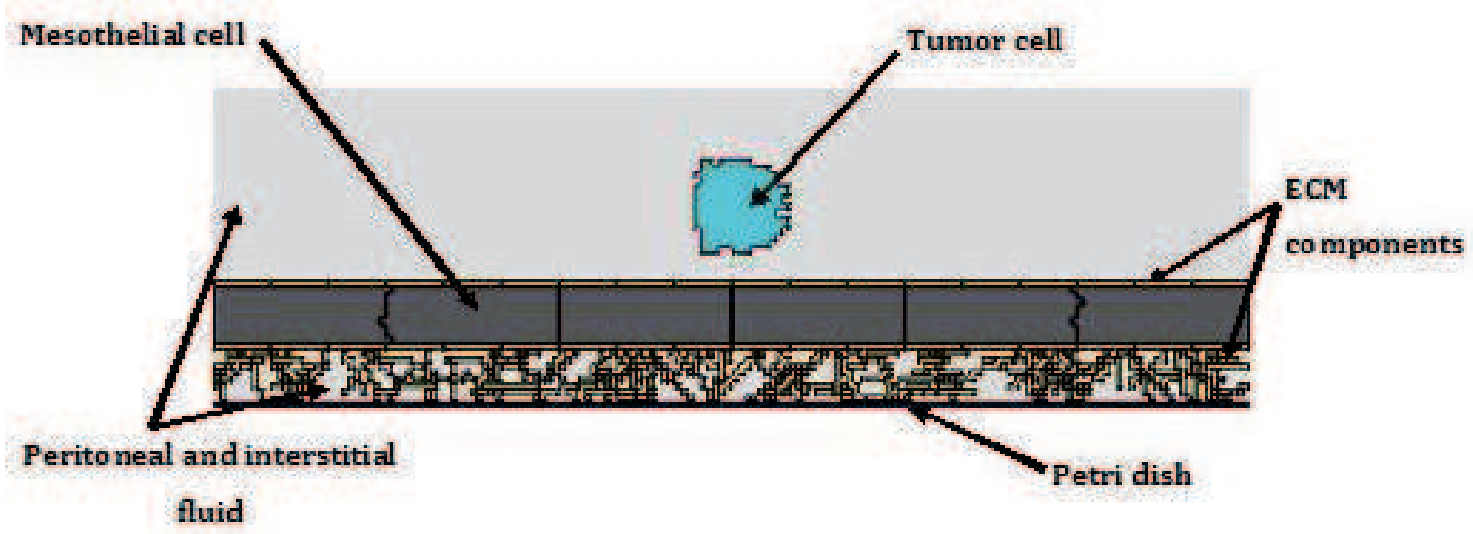

Figure 6: Initial condition of simulated single tumor cell mesothelial invasion and description of the symbols used in the model.

Table II gives the cellular motilities $T_{\tau}$, and the prescribed dimensions and the relative elasticity values (the $\lambda_{\tau}$ and $\gamma_{\tau}$ of Eq. 3.3) for cancer and mesothelial cells. The latters, as outlined by the experiments, are anchored to the ECM fibers, form a continuous and stable pavement and barely move from their original position, maintaining their characteristic shape: conversely, the tumor cell is subjected to dramatic surface and volume alterations and remodeling, since it has the ability to spread on and to suddenly cross the monolayer through gaps created by the retraction of mesothelial cells. These considerations lead to:

$$
\begin{aligned}
& T_{c}>T_{m}, \\
& \lambda_{c}<\lambda_{m}, \\
& \gamma_{c}<\gamma_{m} .
\end{aligned}
$$

TABLE I

CELL TYPES USED IN SIMULATIONS

\begin{tabular}{|c|c|}
\hline \hline Symbol & Description \\
\hline \hline$\tau_{c}$ & Tumor \\
$\tau_{m}$ & Mesothelium \\
$\tau_{f}$ & Peritoneal and Interstitial Fluid \\
$\tau_{e}$ & Extracellular Matrix Components \\
$\tau_{s}$ & Petri Dish \\
\hline \hline
\end{tabular}

The cancer cell's motion is initially directed in response to chemotactic factors released by mesothelial cells and ECM molecules, whose local level of concentration $h$ is controlled by the following PDE:

$$
\frac{\partial h}{\partial t}=D_{c h} \nabla^{2} h-k_{c h} h+\theta_{c h}(\mathbf{x})
$$


TABLE II

PARAMETERS GOVERNING THE MODEL OF SINGLE CELL INVASION

\begin{tabular}{|c|c|c|}
\hline \hline Parameter & Description & Value \\
\hline \hline$T_{c}$ & Motility of $\tau_{c}$ & 20 \\
$T_{m}$ & Motility of $\tau_{m}$ & 10 \\
\hline$V_{c}$ & Target Volume of $\tau_{c}$ & $175\left(\mathrm{in} \mathrm{px}^{2}\right)$ \\
$V_{m}$ & Target Volume of $\tau_{m}$ & $243\left(\mathrm{in} \mathrm{px}^{2}\right)$ \\
$S_{c}$ & Target Surface of $\tau_{c}$ & $70(\mathrm{in} \mathrm{px})$ \\
$S_{m}$ & Target Surface of $\tau_{m}$ & $72(\mathrm{in} \mathrm{px})$ \\
\hline$\lambda_{c}$ & Volume Elasticity of $\tau_{c}$ & 3 \\
$\lambda_{m}$ & Volume Elasticity of $\tau_{m}$ & 10 \\
$\gamma_{c}$ & Surface Elasticity of $\tau_{c}$ & 5 \\
$\gamma_{m}$ & Surface Elasticity of $\tau_{m}$ & 10 \\
\hline$J_{c, f}$ & Adhesion between $\tau_{c}$ and $\tau_{f}$ & 5 \\
$J_{m, f}$ & Adhesion between $\tau_{m}$ and $\tau_{f}$ & -1 \\
$J_{e, f}$ & Adhesion between $\tau_{e}$ and $\tau_{f}$ & -1 \\
$J_{c, e}$ & Adhesion between $\tau_{c}$ and $\tau_{e}$ & -1 \\
$J_{c, m}$ & Adhesion between $\tau_{c}$ and $\tau_{m}$ & -15 \\
$J_{m, e}$ & Adhesion between $\tau_{m}$ and $\tau_{e}$ & -10 \\
$J_{m, m}$ & Adhesion between $\tau_{m}$ and $\tau_{m}$ & 30 \\
$J_{c, s}$ & Adhesion between $\tau_{c}$ and $\tau_{s}$ & $0.2\left(\mathrm{MCS}^{-1}\right)$ \\
\hline$\mu_{c}$ & Chemotactic Force on $\tau_{c}$ & \\
\hline$D_{M M P}$ & MMPs Diffusion Rate & $0.02\left(\mathrm{px}^{2} \mathrm{MCS}^{-1}\right)$ \\
$k_{M M P}$ & MMPs Decay Rate & $0.01\left(\mathrm{MCS}^{-1}\right)$ \\
$\theta_{M M P}$ & MMPs Secretion Rate & $0.25\left(\mathrm{MCS}^{-1}\right)$ \\
$D_{c h}$ & Chemoattractant Diffusion Rate & $0.1\left(\mathrm{px}^{2} \mathrm{MCS}^{-1}\right)$ \\
$k_{c h}$ & Chemoattractant Decay Rate & $0.001\left(\mathrm{MCS}^{-1}\right)$ \\
$\theta_{c h}$ & Chemoattractant Secretion Rate & 0.2 \\
\hline \hline & & \\
\hline
\end{tabular}

The coefficients of diffusion $D_{c h}$ and decay $k_{c h}$ are assumed homogeneous throughout the simulation domain and constant in time, while $\theta_{c h}(\mathbf{x})$, describing the secretion of the chemoattractant is given by:

$$
\theta_{c h}(\mathbf{x})= \begin{cases}\theta_{c h} & \text { if } \quad \sigma(\mathbf{x}) \in\left(\tau_{m}, \tau_{e}\right) \wedge \sigma\left(\mathbf{x}^{\prime}\right)=\tau_{e} \\ 0 & \text { else }\end{cases}
$$

being $\mathbf{x}^{\prime}$ one of the neighbors of a generic lattice site $\mathbf{x}$. The release of the chemotactic signals from the virtual mesothelial cells is inhibited after the adhesion of the tumor to the layer $\left(\theta_{c h}(\mathbf{x})=0\right.$ if $\sigma(\mathbf{x})=\tau_{m}$ ), while continues from the ECM fibers. The relative chemotaxis coefficient $\mu_{c}$ of Eq. (3.5) is obviously not null only for the cancer cell.

Table II also gives the initial values of the adhesion energies $J$ 's. They are spatially constant, meaning that coupling strengths are uniformly distributed over the cells' surfaces and modeling an homogeneous localization and expression of the cell adhesion molecules over the cellular membranes. The initial hierarchy of the J's is important to maintain the structure of the mesothelial layer rigid, surrounded by the pericellular matrix and fixed on the submesothelial ECM fibers. In particular $J_{m, m}$ is very low, mimicking a strong adhesion energy between the mesothelial cells, consequence of the high level of expression principally of $\mathrm{N}$-cadherins.

The malignant cell's attachment via $\beta 1$-integrin and CD44 molecule to the layer is modeled decreasing both $J_{c, e}$ and $J_{c, m}$ : this over-expression of integrin subunits causes the activation and 


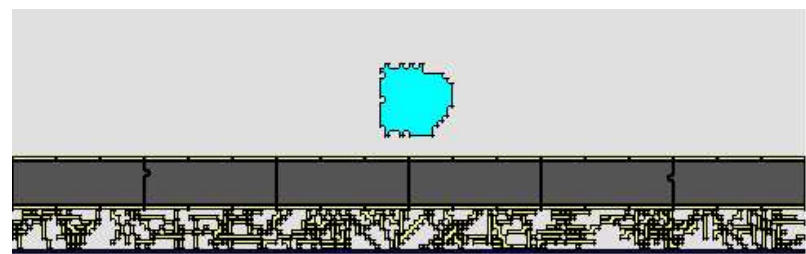

(A)

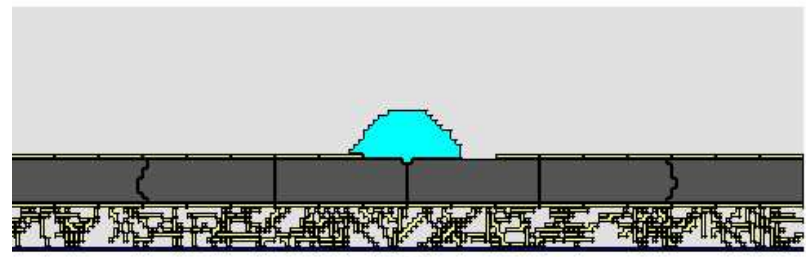

(C)

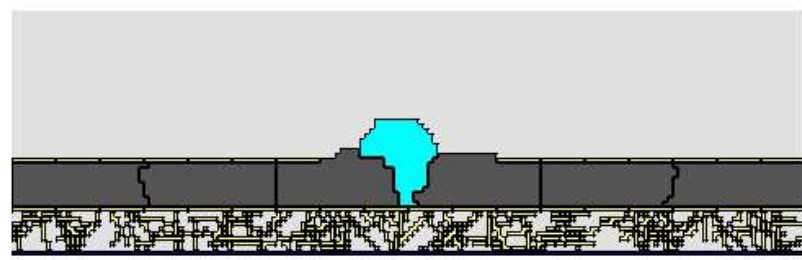

(E)

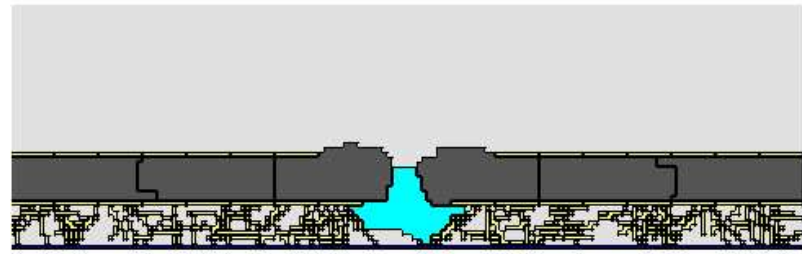

(G)

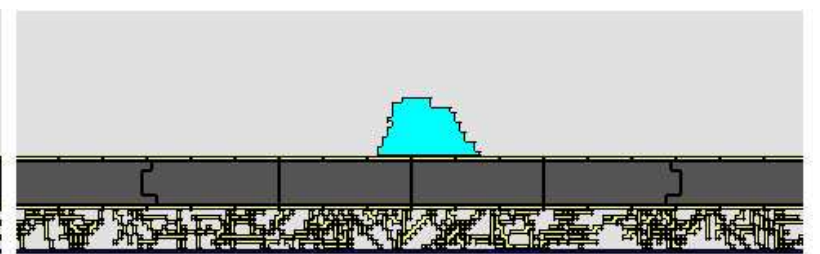

(B)

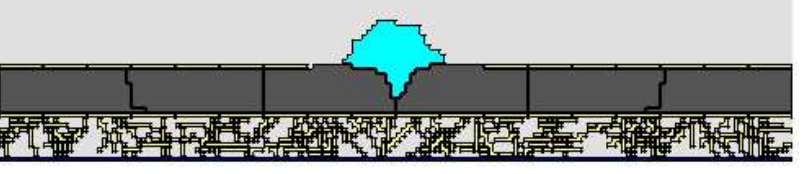

(D)

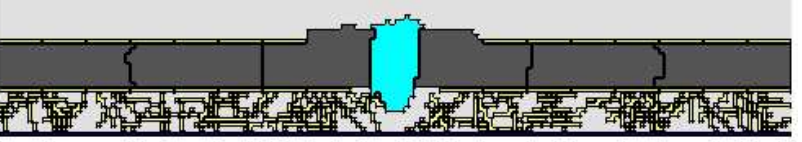

(F)

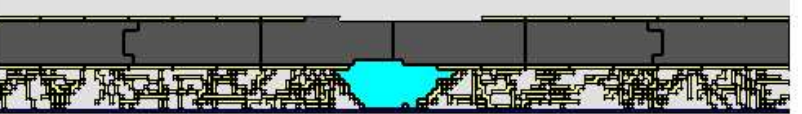

(H)

Figure 7: Simulated single cell transmesothelial migration at 0, 200, 400, 700, 800, 950, 1100, and 1200 MCS. In (B) the cell touches the mesothelial layer covered by the pericellular matrix and starts spreading over it. In (C) the production of MMPs starts degrading the pericellular layer. In (D) the cancer cell induces the loosening of the adhesion bonds between the epithelial cells that detach so that the cell progressively penetrates the layer. After the cell has migrated to the opposite side of the layer $(\mathrm{G})$, the simulated mesothelium has closed back $(\mathrm{H})$, and the tumor cell is considered completely infiltrated.

the release of different type of MMPs [33], whose total amount $\left(h_{M M P}\right)$ and evolution is described by

$$
\frac{\partial h_{M M P}}{\partial t}=D_{M M P} \nabla^{2} h_{M M P}-k_{M M P} h_{M M P}+\theta_{M M P}(\mathbf{x}),
$$

where, as before, $D_{M M P}$ and $k_{M M P}$ are, respectively, the diffusion and degradation rate, and

$$
\theta_{M M P}(\mathbf{x})= \begin{cases}\theta_{M M P} & \text { if } \sigma(\mathbf{x})=\tau_{t} \wedge \sigma\left(\mathbf{x}^{\prime}\right)=\tau_{e} \\ 0 & \text { else }\end{cases}
$$


for $\mathbf{x}, \mathbf{x}^{\prime}$ neighboring pixels. The secretion will be inhibited as the malignant cell attaches to the virtual Petri dish $\left(\theta_{M M P}=0\right)$.

During the invasion process, the MMPs are capable to degrade both the pericellular and the submesothelial matrix components: to model this biological effect, a lattice site $\mathbf{x}$ within an ECM molecule becomes a generalized pixel of medium when the local level of MMPs $\left(h_{M M P}(\mathbf{x})\right)$ is sufficiently high, reaching the threshold of 2.5. This change is implemented by changing $\tau(\sigma(\mathbf{x}))$ from $\tau_{e}$ to $\tau_{f}$, corresponding to the disruption of the pericellular layer (see Figure 7C).

The activity of tumor MMPs also affects and down-regulates the expression of the cadherins both in cancer and in mesothelial cells, breaking cell-cell adhesion and making the mesothelial cells retract: mathematically $J_{c, m}$ and $J_{m, m}$ increase, and in particular the latter becomes positive. This generates the penetration of the cell in the layer, as shown in Figures 7D-F. The malignant cell, once crossed the layer, loses the capacity to inhibit cadherin signalling ( $J_{c, m}$ and $J_{m, m}$ decrease until their initial values), leading to the reconnection of the junctions between mesothelial cells and between them and the tumor cell, which becomes covered by the monolayer as shown in Figure $7 \mathrm{H}$. All the proposed changes in the adhesion energy values are performed with a unit increment (or decrement) every 10 MCS. The overall simulation lasts 1200 MCS, which, fitting the experimental times, we set to correspond to about $5 \mathrm{~h}$, defining $1 \mathrm{MCS} \approx 15$ seconds. In particular the cancer cell takes time to adhere to the layer and to degrade the ECM components, while the effective mesothelial infiltration is 500 MCS long ( $\approx 2$ hours), see Figures 7D-H.

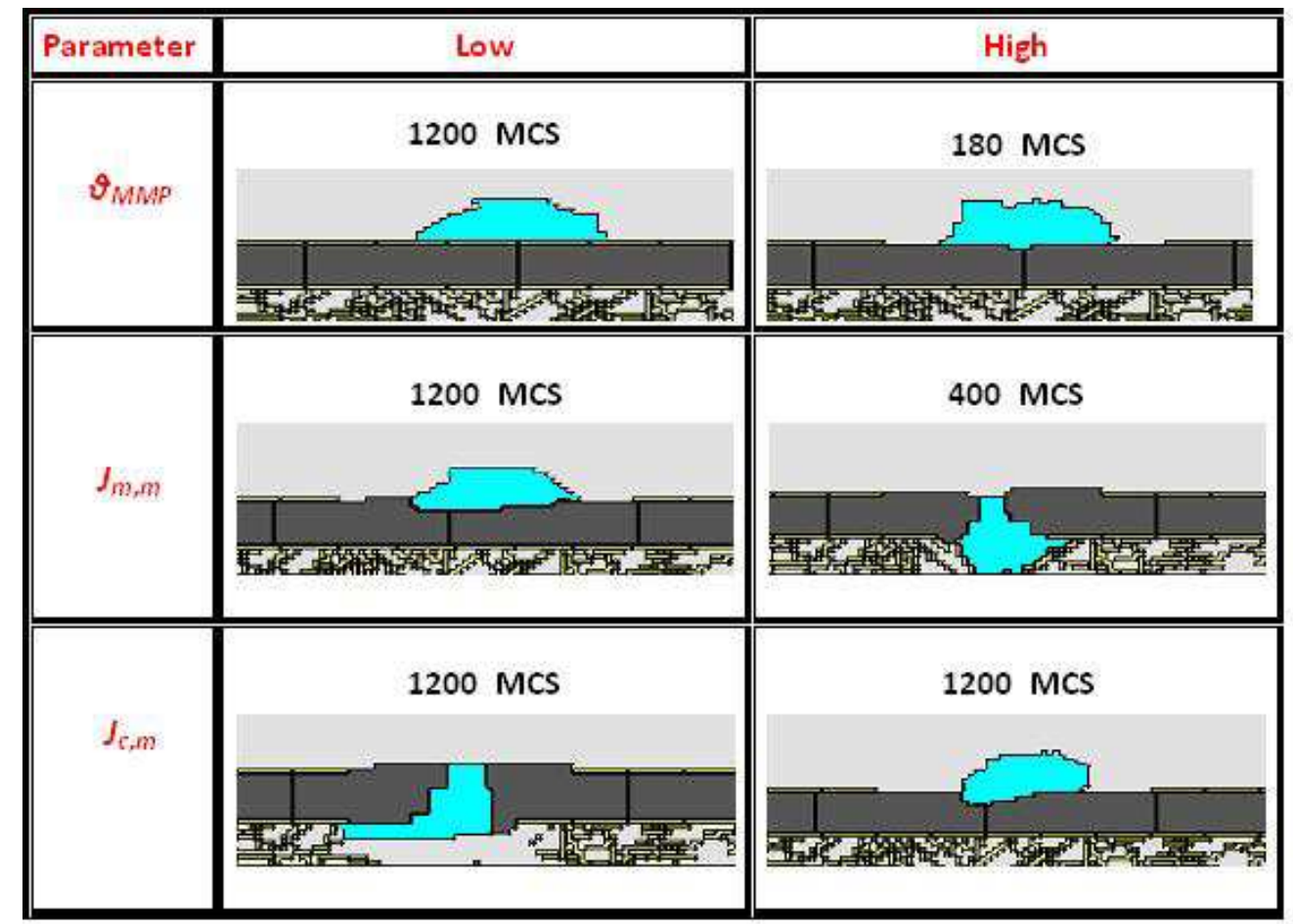

Figure 8: Summary of the simulations of single cell transmesothelial migration run with different parameters. All the other parameters not explicitly cited are the same as in Table II. 
The partial differential equations used for the macroscopical description of the chemoattractant and the proteases fields are solved numerically using a finite difference scheme on lattices that match the CPM grid.

To test our model and to underline how the single terms affect the biological outputs, we ran sets of simulations changing one parameter at a time, while keeping the others fixed as in Table II. The results are summarized in Figure 8.

We have the confirmation that the cancer cells acquire malignant properties when MMPs' expression is strongly up-regulated (with a strong decrease in the transmigration time) and that, in contrast, they become less aggressive when the MMPs' secretion is reduced as they lose the ability to degrade the matrix components. Mathematically, low and high MMPs' releases are referred to their ratio with the threshold value (2.5) characteristic of the transition between an ECM and the medium.

With constant very low $J_{m, m}$ values $\left(J_{m, m} \ll J_{c, m}\right)$, we simulate an over-expression of the $\mathrm{N}$-cadherins connecting the mesothelial cells: this prevents the invasion of the tumor, which can only adhere at the top of the layer: instead, higher values of $J_{m, m}\left(J_{m, m} \gg J_{c, m}\right)$ facilitate and accelerate its transmigration. Low values of $J_{c, m}\left(J_{c, m} \ll J_{c, e}, J_{m, m}\right)$ model the fact that the ovary carcinoma cell may become part of the layer, since it is an epithelial-type cell and can express the same cell adhesion molecules as the mesothelial cells. In contrast, for high values of $J_{c, m}$ $\left(J_{c, m} \gg J_{c, e}, J_{m, m}\right)$ the tumor cell has few adhesion point with the virtual mesothelium and doesn't activate the downstream cadherin-signals needed for the invasion program.

\section{Simulation of Multi-Cellular Spheroid Invasion}

Figure 8 shows a typical simulation of a time-sequence spheroid invasion of a mesothelial layer: the initial condition are the same as in Figure 6 but the tumor mass is formed by 10 virtual cells. The features of the model are structurally analogous to those of the previous section and the simulations last $12000 \mathrm{MCS}(\approx 2$ day), which lets us not consider cancer cell proliferation. The only differences are the parameters explicitly cited in Table III and the fact that we also take into account the sedimentation of the spheroid due to gravity, which directs, along with the classical chemotactic cues, the motion of the tumor mass towards the layer.

TABLE III

PARAMETERS GOVERNING THE MODEL OF SPHEROID INVASION

\begin{tabular}{|c|c|c|}
\hline \hline Parameter & Description & Value \\
\hline \hline$S_{c}$ & Target Surface of $\tau_{c}$ & 56 (in px) \\
\hline$\lambda_{c}$ & Volume Elasticity of $\tau_{c}$ & 5 \\
\hline$J_{c, c}$ & Adhesion between $\tau_{c}$ and $\tau_{c}$ & -1 \\
$J_{c, m}$ & Adhesion between $\tau_{c}$ and $\tau_{m}$ & 9 \\
\hline$\theta_{M M P}$ & MMPs Secretion Rate & $0.3\left(\mathrm{MCS}^{-1}\right)$ \\
\hline$g$ & Gravitational Potential & 3 \\
\hline \hline
\end{tabular}




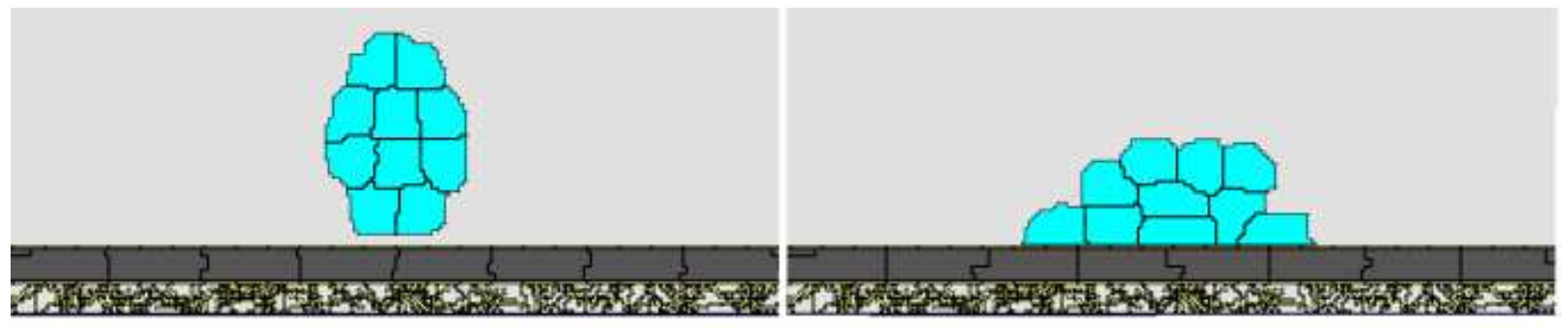

(A)

(B)

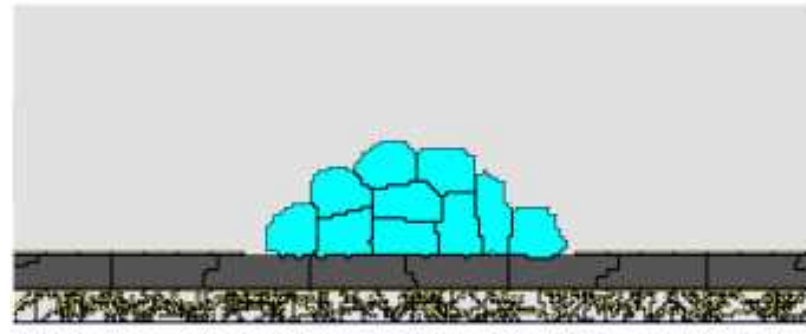

(C)

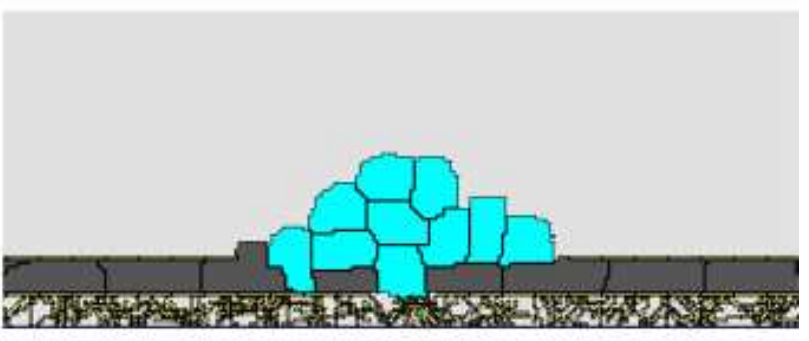

(D)

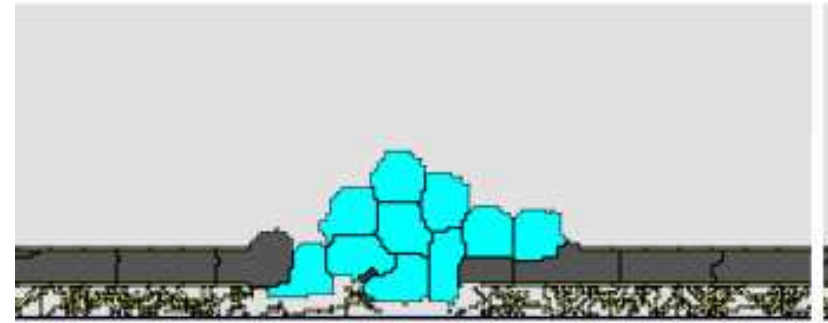

(E)

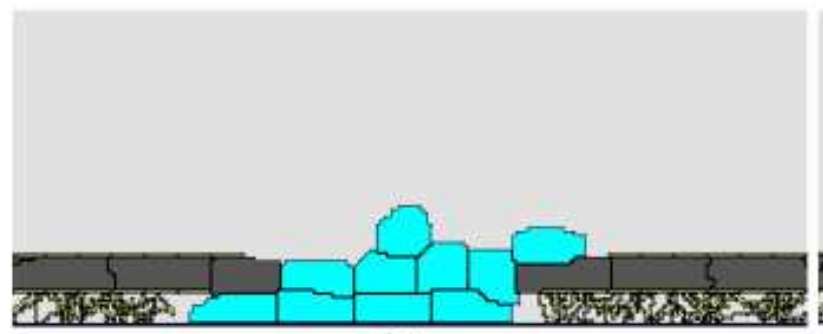

(G)

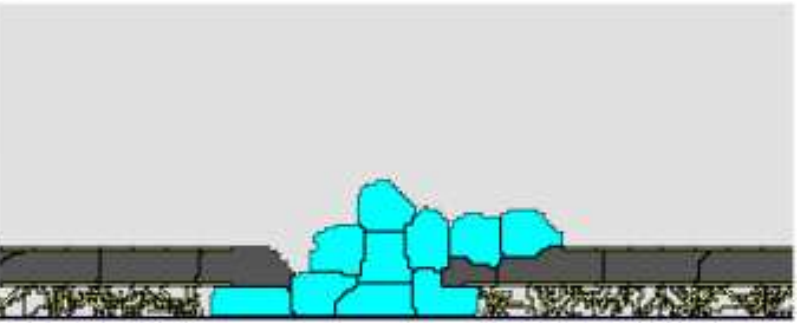

(F)

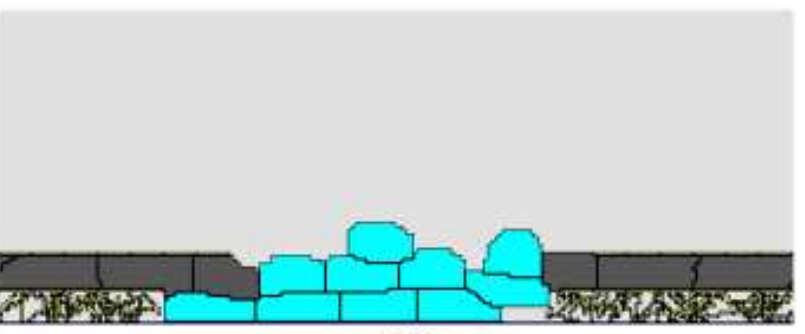

(H)

Figure 9: Simulated multi-cellular spheroid transmesothelial migration at 0, 2000, 4000, 6000, 7500, 9000, 10500, 12000 MCS. The tumor mass adheres to the layer (B) and the subsequent expression of MMPs degrades the ECM components and breaks the junctions between the mesothelial cells (C) and (D). The disseminated spheroid invades the virtual mesothelium through different foci of adhesion, causing the apoptosis of the mesothelial cells nearby (E), $(\mathrm{F})$ and $(\mathrm{G})$. At the end of the simulation $(\mathrm{H})$, the cancer mass has overtaken a large area of the monolayer and starts the metastatic outgrowth. 
We use lower $S_{c}$ and higher $\gamma_{c}$ since the tumor cells, acquiring a spheroid morphology, are tightly packed and with difficulty change their shape or remodel, remaining almost round with a constant volume/surface ratio. The strong homotypic interactions, regulated by the expression of $\beta 1$-integrins, E-cadherins, and by other intercellular mechanisms are modeled by an initial negative $J_{c, c}$, which increases as the cancer aggregate starts disseminating over the monolayer. The higher value of $J_{c, m}$, with respect to the case of the single cell, represents the reported lower overall spheroid-mesothelium adhesion [19]. The longer time needed by the tumor mass to attach to ECM components is modeled with a slow decrease ( 0.01 instead of 1 each $10 \mathrm{MCS})$ of the relative bond energy $J_{c, e}$ (see Figure 9B). This reflects a low expression and avidity of cancer integrin subunits and physical and geometrical constraints limiting its ability to spread on anchoring surfaces.

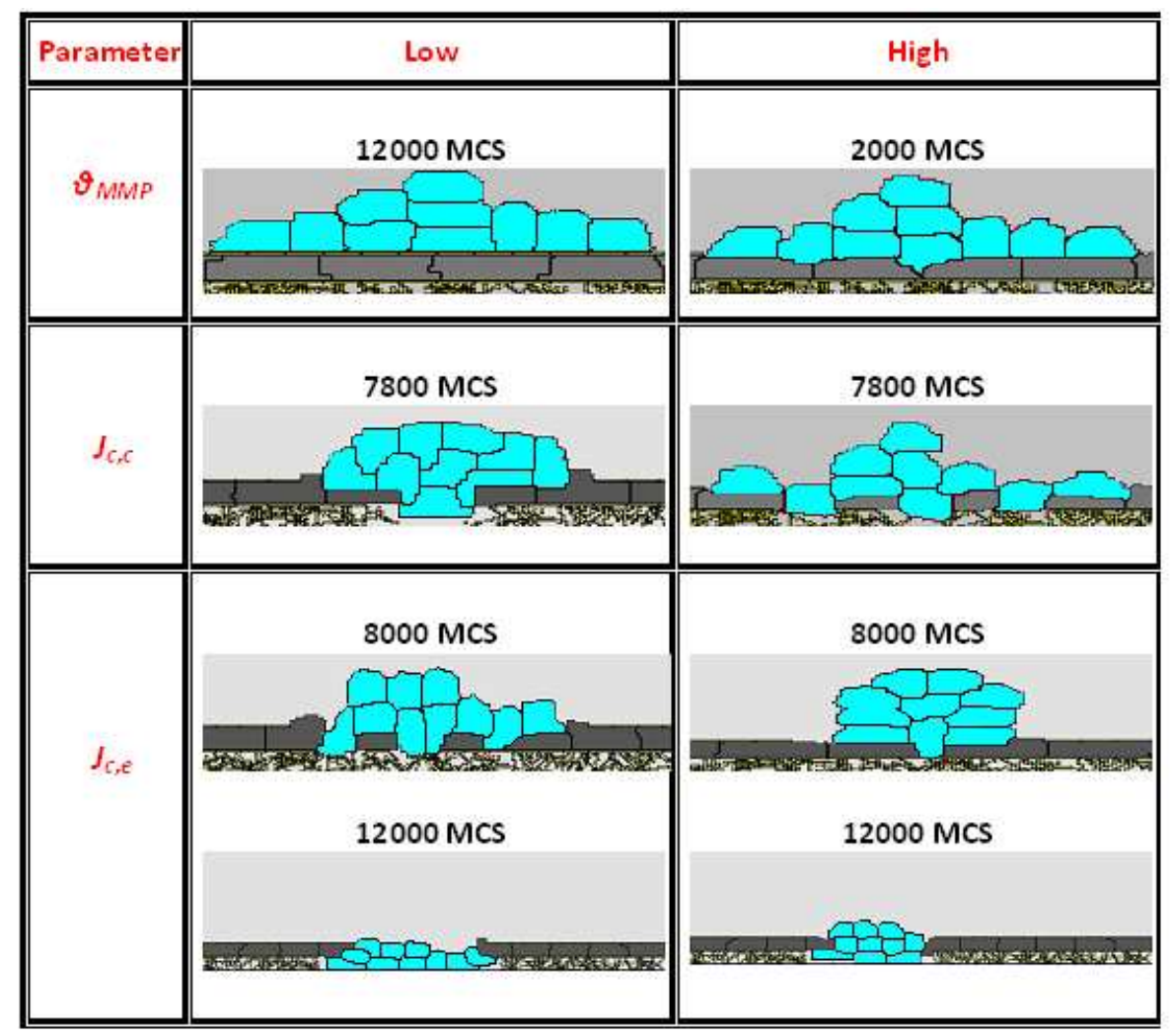

Figure 10: Summary of the simulations of multi-cellular spheroid invasion run with different parameters. All the parameters not explicitly cited are the same of Table II and III.

The spatial and temporal evolution of the proteases' field are the same as in the case of the single cells, but the total amount of the MMPs secreted is higher (see Table III), not only for the increased tumor mass but also because the cancer cells, upon acquiring a spheroid morphology, over-express the different types of proteases and have an enhanced invasive potential, as shown in Figures 9C and D. Since experimental evidences show that generally the mesothelial layer disaggregates and retracts and that isolated mesothelial cells beneath the cancer cells die, in the simulation the target volume $V_{m}$ of the virtual mesothelial cells surrounded by the malignant mass (30 px 
of common surface) is set to decrease, 1 px each MCS, until a limit value, $80 \mathrm{px}$, for which they undergo apoptosis. This generates the disruption of the mesothelial layer shown in Figure 9E-G .

At the end of the simulated process, the multiple invading foci merge and are able to overtake the well, leaving little of the mesothelial cell monolayer intact. It is clear that the malignant cells disseminate not only in the same plane, but also above and below the virtual mesothelium, and degrade large part of the ECM components, as shown in Figure 9H.

Also in the case of the multi-cellular spheroids an increase in MMPs' release causes an acceleration in the overall metastatic programm (top of Figure 10).

Low $J_{c, c}$ values $\left(J_{c, c} \ll J_{c, m}, J_{c, e}\right)$ model the preference of the spheroid cells to maintain their strong homotypic connections, rather than disseminate and establish heterotypic interactions with mesothelial cells or matrix components: in this case the spheroids have also the tendency to form only a single focus of invasion.

In contrast, for high $J_{c, c}\left(J_{c, c} \gg J_{c, m}, J_{c, e}\right)$, the cancer cells quickly detach from the core of the aggregate and disseminate on a larger area of the mesothelial layer, invading in different sites (middle of Figure 10). A similar phenomenology can be obtained by forcing an over-expression of $\beta 1$-integrins in the tumor mass by strongly decreasing $J_{c, e}\left(J_{c, e} \ll J_{c, m}, J_{c, c}\right)$, bottom of Figure 10.

\section{Conclusions}

We have reproduced conventional in vitro ovarian cancer transmigration assays, focusing on single cells and cell aggregates. We have shown that the simulations are in good agreement with experimental evidences and demonstrated that changes in the expression of selected adhesive molecules, along with the release of tumor matrix metalloproteinases and the consequent degradation of ECM components are the key players of the overall process. The model, supporting experimental studies, shows that mesothelial invasion by single cells is more conservative. Isolated cells can rapidly change their shape to cross the mesothelium through gaps opened by the activity of the MMPs, but, once they have passed, the mesothelial junctions are recovered and the continuity of the layer restored. Instead, cancer spheroids, completely overtaking larger areas of the mesothelium, form dramatic foci of invasion, induce apoptosis of the detached part of the mesothelium, and start their metastatic outgrowth.

This work is in agreement with the observation that high levels of MMPs in tumor correlate with poor prognosis [8], and that the overall transmesothelial migration is time-regulated by the expression and the interplay of cell-cell and cell-matrix adhesion molecules.

In our ongoing work we are refining and validating our computational model by obtaining experimentally derived values for the model parameters and by comparing our simulation results quantitatively to time-lapse videomicroscopy experiments. We also plan to insert in the model structure the sub-cellular pathways governing the expression and the activity of adhesion molecules and the secretion of the matrix metalloproteinases, and to relate them to the cell-level phenomenology. In particular, it seems very promising to study the functions of the molecule CD157 [12] which 
seems to play a major role in mesothelial invasion.

Another interesting development is to extend the model to reproduce the in vivo ovarian peritoneal metastasis, for example by adding part of the connective tissue and of the vasculature below the mesothelium, and to test if it is regulated by the same intra- and inter-cellular interactions.

\section{References}

[1] N. Ahmed, E. W. Thomson, M. A. Quinn. Epithelial - mesenchymal interconversions in normal ovarian surface epithelium and ovarian carcinomas: an exception to the norm. J. Cell. Physiol., 213 (2007), 581-588.

[2] J. Ahmedin, T. Murray, A. Samuels, A. Ghafoor, E. War, M. J. Thun. Cancer statistics. Cancer J. Clin., 53 (2003), 5-26.

[3] K. M. Burleson, R. C. Casey, K. M. Skubitz, E. Pambuccian, T. R. Oegema Jr, A. P. Skubitz. Ovarian carcinoma ascites spheroids adhere to extracellular matrix components and mesothelial cell monolayers. Gynec. Oncol., 93 (2004), 170-181.

[4] K. M. Burleson, M. P. Boente, S. E. Parmabuccian, A. P. Skubitz. Ovarian carcinoma spheroids disaggregate on type I collagen and invade live mesothelial cell monolayers. Clin. Exp. Metastasis, 21 (2004), 685-697.

[5] K. M. Burleson, M. P. Boente, S. E. Parmabuccian, A. P. Skubitz. Disaggregation and invasion of ovarian carcinoma ascites spheroids. J. Transl. Med., 4 (2006),1-16.

[6] S. A. Cannistra. Cancer of the ovary. N. Engl. J. Med. 329 (1993), 1550-1559.

[7] R. C. Casey, K. M. Burleson, K. M. Skubitz, S. E. Parmabuccian, T. J. Oegema, L. E. Ruff,

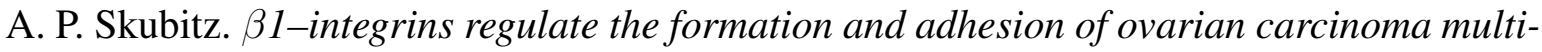
cellular spheroids. Am. J. Pathol., 159 (2001), 2071-2080.

[8] M. Egeblad, Z. Werb. New functions for the matrix metalloproteinases in cancer progression. Nature, 2 (2002), 2071-2080.

[9] K. M. Feeley, M. Wells. Precursor lesions of ovarian epithelial malignancy. Histopathology, 38 (2001), 87-95.

[10] A. Feki, P. Berardi, G. Bellingan, A. Major, K. H. Krause, P. Petignat. Dissemination of intraperitoneal ovarian cancer: Discussion of mechanisms and demonstration of lymphatic spreading in ovarian cancer model. Crit. Rev. Oncol. Hematol., 72 (2009), 1-9.

[11] D. A. Fishman, Y. Liu, S. M. Ellerbroek, M. S. Stack. Lysophosphatidic acid promotes Matrix Metalloproteinase (MMP) activation and MMP-dependent invasion in ovarian cancer cells. Cancer Res., 61 (2001), 3194-3199. 
[12] A. Funaro, E. Ortolan, P. Bovino, N. Lo Buono, G. Nacci, E. Parrotta, E. Ferrero, F. Malavasi. Ectoenzymes and innate immunity: the role of human CD157 in leukocyte trafficking. Front. Biosci., 14 (2009), 929-943. Review.

[13] J. A. Glazier, A. Balter, N. J. Poplawski. Magnetization to morphogenesis: a brief history of the Glazier-Graner-Hogeweg model. In A. R. A. Anderson, M. A. J. Chaplain, and K. A. Rejniak editors, Single-Cell-Based Models in Biology and Medicine, Mathematics and Biosciences in Interactions, pages 79-106. Birkaüser, 2007.

[14] J. A. Glazier, F. Graner, Simulation of the differential adhesion driven rearrangement of biological cells. Physical. Rev. E., 47 (1993), 2128-2154.

[15] F. Graner, J. A. Glazier. Simulation of biological cell sorting using a two-dimensional extended Potts model. Phys. Rev. Letters, 69 (1992), 2013-2017.

[16] H. G. E. Hentschel, T. Glimm, J. A. Glazier, S. A. Newman. Dynamical mechanisms for skeletal pattern formation in the vertebrate limb. Proc. R. Soc. Lond. B (2004), 1713-1722.

[17] J. M. Kelm, N. E. Timmins, C. J. Brown, M. Fussenegger, L.K. Nielsen. Method for generation of homogeneous ulticellular tumor spheroids applicable to a wide variety of cell types. Biotechnol. Bioeng., 83 (2003), 173-180.

[18] H. A. Kenny, S. Kaur,L. M. Coussens, E. Lengyel. The initial steps of ovarian cancer cell metastasis are mediated by MMP-2 cleavage of vitronectin and fibronectin. J. Clin. Invest. 118 (2008), 1367-1379.

[19] K. Lessan, D. J. Aguiar, T. J. Oegema, L. Siebenson, A. P. Skubitz. CD44 and B1-integrin mediate ovarian carcinoma cell adhesion to peritoneal mesothelial cells. Am. J. Pathol., 154 (1999), 1525-1537.

[20] J. S. Lowergrub, H. B. Frieboes, F. Jin, Y. L. Chuang, X. Li, P. Macklin, S. M. Wise, V. Cristini. Nonlinear modeling of cancer: bridging the gap between cells and tumor. Nonlinearity. In press.

[21] A. F. M. Marée, V. A. Grieneisen P. Hogeweg. The Cellular Potts Model and biophysical properties of cells, tissues and morphogenesis. In A. R. A. Anderson, M. A. J. Chaplain, and K. A. Rejniak editors, Single-Cell-Based Models in Biology and Medicine, Mathematics and Biosciences in Interactions, pages 107-136. Birkaüser, Basel, Switzerland, 2007.

[22] R. M. H. Merks, J. A. Glazier. Dynamic mechanisms of blood vessel growth. Institute of Physics Publishing, 19 (2006), C1-C10.

[23] R. M. H. Merks, J. A. Glazier, A. Balter, N. J. Poplawski, M. Swat. The Glazier-GranerHogeweg model: extensions, future directions, and opportunities for further study. Mathematics and Biosciences in Interaction (2007), 151-167. 
[24] R. M. H. Merks, J. A. Glazier. A cell-centered approach to developmental biology. Physica. A., $352(2005), 113-130$.

[25] S. E. Mutsaers. Mesothelial cells: their structure, function and role in serosal repair. Respirology, 7 (2002), 171-191.

[26] H. Naora, D. J. Montell. Ovarian cancer metastasis: integrating insights from disparate model organisms. Nat. Rev. Cancer, 5 (2005), 355-366.

[27] M. J. Niedbala, K. Crickard, R. J. Bernacki. Interactions of human ovarian tumor cells with human mesothelial cells grown on extracellular matrix. An in vitro model system for studying tumor cell adhesion and invasion. Exp. Cell. Res., 160 (1985), 499-513.

[28] N. J. Poplawski, A. Shirinifard, M. Swat, J. A. Glazier. Simulation of single-species bacterical-biofilm growth using the Glazier-Graner-Hogeweg model and the CompuCell3D modeling environment. Math. Biosci. Eng., 5 (2008), 355-388.

[29] S. Patel, P. Madan, S. Getsios, M. A. Bertrand, C. D. Maccalman. Cadherin switching in ovarian cancer progression. Int. J. Cancer., 106 (2003), 172-177.

[30] L. Preziosi, A. Tosin. Multiphase and multiscale trends in cancer modelling. Math. Model Nat. Phenomena, 4 (2009), 1-11.

[31] M. L. Puiffe, C. La Page, A. Filali-Mouhim, M. Zietarska, V. Ouellet, P. N. Toniny, M. Chevrette, D. M. Provencher, A. M. Mes-Masson. Characterization of ovarian cancer ascites on cell invasion, proliferation, spheroid formation, and gene expression in an in vitro model of epithelial ovarian cancer. Neoplasia, 9 (2007), 820-829.

[32] N. J. Savill, P. Hogeweg. Modelling morphogenesis: from single cells to crawling slugs. J. Theor. Biol., 184 (1997), 118-124.

[33] K. Sawada, A. K. Mitra, A. Reza Radjabi, V. Bhaskar, E. O. Kistner, M. Tretiakova, S. Jagadeeswaran, A. Montag, A. Becker, H. A. Kenny, M. E. Peter, V. Ramakrishnan, S. D. Yamada, E. Lengyel. Loss of E-cadherin promotes ovarian cancer metastasis via $\alpha 5$-integrin, which is a therapeutic target. Cancer Res., 68 (2008), 2329-2339.

[34] M. Sawada, J. Shii, H. Akedo, O. Tanizawa. An experimental model for ovarian tumor invasion of cultured mesothelial cell monolayer. Lab. Invest., 70 (1994), 333-338.

[35] M. Scianna, R. M. H. Merks, L. Preziosi, E. Medico. Individual cell-based models of cell scatter of ARO and MLP-29 cells in response to hepatocyte growth factor. J. Theor. Biol. 260 (2009), 151-160.

[36] K. Shield, M. L. Ackland, N. Ahnmed, G. E. Rice. Multicellular spheroids in ovarian cancer metastases: Biology and pathology. Gynec. Oncol., 113 (2008), 143-148. 
[37] K. Shield, C. Riley, M. A. Quinn, G. E. Rice, M. L. Ackland, N. Ahnmed. $\alpha 2 \beta 1$-integrin affects metastatic potential of ovarian carcinoma spheroids by supporting disaggregation and proteolysis. J. Carcinog., 6 (2007), 6-11.

[38] P. N. Skubitz, R. C. Bast Jr, E. A. Wayner, P. C. Letourneau, M. S. Wilke. Expression of $\alpha 6$ and $\beta 4$ integrins in serous ovarian carcinoma correlates with expression of the basement membrane protein laminin. Am. J. Pathol., 148 (1996), 1445-1461.

[39] K. Sundfeldt. Cell-cell adhesion in the normal ovary and ovarian tumors of epithelial origin; an exception to the rule. Molecular and Cellular Endocrinology, 202 (2003), 89-96.

[40] S. Yung, F. K. Li, T. M. Chan. Peritoneal mesothelial cell culture and biology. Perit. Dial. Int., 26 (2006), 162-173.

[41] F. Wang, J. So, S. Reierstad, D. A. Fishman. Vascular endothelial growth factor regulated ovarian cancer invasion and migration involves expression and activation of matrix metalloproteinases. Int. J. Cancer, 118 (2006), 879-888.

[42] H. S. Wang, Y. Hung, C. H. Su, S. T. Peng, Y. J. Guo, M. C. Lai MC. CD44 cross-linking induces integrin-mediated adhesion and transendothelial migration in breast cancer cell line by up-regulation of LFA-1 ( $\alpha L \beta 2)$ and VLA-4 ( $\alpha 4 \beta 1)$. Exp. Cell. Res., 304 (2005), 116-126.

[43] Y. Zhu, K. Sunfeldt. Tight junction formation in epithelial ovarian adenocarcinoma. Acta Obstetricia et Gynecologica, 86 (2007), 1011-1019. 